\title{
Sensitivity Minimization by Stable Controllers: An Interpolation Approach for Suboptimal Solutions
}

\author{
Suat Gumussoy and Hitay Özbay
}

\begin{abstract}
Weighted sensitivity minimization is studied within the framework of strongly stabilizing (stable) $\mathcal{H}^{\infty}$ controller design for a class of infinite dimensional systems. This problem has been solved by Ganesh and Pearson, [8], for finite dimensional plants using Nevanlinna-Pick interpolation. We extend their technique to a class of unstable time delay systems. Moreover, we illustrate suboptimal solutions, and their robust implementation.
\end{abstract}

\section{INTRODUCTION}

In this note we study sensitivity minimization problem for a class of infinite dimensional systems. The goal is to minimize the $\mathcal{H}^{\infty}$ norm of the weighted sensitivity by using stable controllers from the set of all stabilizing controllers for the given plant. This problem is a special case of strongly stabilizing (i.e. stable) controller design studied earlier, see for example [2], [3], [4], [5], [10], [13], [14], [16], [18], [19], [20], [21], [25], [27], [28], and their references for different versions of the problem. The methods in [1], [8] give optimal (sensitivity minimizing) stable $\mathcal{H}^{\infty}$ controllers for finite dimensional SISO plants. Other methods provide sufficient conditions to find stable suboptimal $\mathcal{H}^{\infty}$ controllers. As far as infinite dimensional systems are concerned, [9], [23] considered systems with time delays.

In this paper, the method of [8] is generalized for a class of time-delay systems. The plants we consider may have infinitely many right half plane poles. Optimal and suboptimal stable $\mathcal{H}^{\infty}$ controllers are obtained for the weighted sensitivity minimization problem using the Nevanlinna-Pick interpolation.

It has been observed that (see e.g. [8], [12]) the Nevanlinna-Pick interpolation approach used in these papers lead to stable controllers with "essential singularity" at infinity. This means that the controller is non-causal, i.e. it contains a time advance, as seen in the examples. In this note, by putting a norm bound condition on the inverse of the weighted sensitivity we obtain causal suboptimal controllers using the same interpolation approach. This extra condition also gives an upper bound on the $\mathcal{H}^{\infty}$ norm of the stable controller to be designed. Another method for causal suboptimal controller design is a rational proper function search in the set of all suboptimal interpolating functions. This method is also illustrated with an example.

This work was supported in part by the European Commission (contract no. MIRG-CT-2004-006666) and by TÜBİTAK (grant no. EEEAG105E156).

S. Gumussoy is with MIKES Inc., Akyurt, Ankara, TR-06750, Turkey, suat.gumussoy@mikes.com.tr

H. Özbay is with Dept. of Electrical \& Electronics Eng., Bilkent University, Ankara TR-06800, Turkey, hitay@bilkent.edu.tr
The problem studied in the paper is defined in Section II. Construction procedure for optimal strongly stabilizing $\mathcal{H}^{\infty}$ controller is given in Section III. Derivation of causal suboptimal controllers is in Section IV. In Section V we give an example illustrating the methods proposed here for unstable time delay systems. Concluding remarks are made in Section VI.

\section{Problem Definition}

Consider the standard unity feedback system with singleinput-single-output plant $P$ and controller $C$. The sensitivity function for this feedback system is $S=(1+P C)^{-1}$. We say that the controller stabilizes the plant if $S, C S$ and $P S$ are in $\mathcal{H}^{\infty}$. The set of all stabilizing controllers for a given plant $P$ is denoted by $\mathcal{S}(P)$, and we define $\mathcal{S}_{\infty}(P)=\mathcal{S}(P) \cap \mathcal{H}^{\infty}$ as the set of all strongly stabilizing controllers.

For a given minimum phase filter $W(s)$ the classical weighted sensitivity minimization problem (WSM) is to find

$$
\gamma_{\mathrm{o}}=\inf _{C \in \mathcal{S}(P)}\left\|W(1+P C)^{-1}\right\|_{\infty} .
$$

When we restrict the controller to the set $\mathcal{S}_{\infty}(P)$ we have the problem of weighted sensitivity minimization by a stable controller (WSMSC): in this case the goal is to find

$$
\gamma_{\mathrm{ss}}=\inf _{C \in \mathcal{S}_{\infty}(P)}\left\|W(1+P C)^{-1}\right\|_{\infty}
$$

and the corresponding optimal controller $C_{\mathrm{ss}, \mathrm{opt}} \in \mathcal{S}_{\infty}(P)$.

The class of plants to be considered here are in the form

$$
P(s)=\frac{M_{n}(s)}{M_{d}(s)} N_{o}(s)
$$

where $M_{n}, M_{d}$ are inner and $N_{o}$ is outer. We will assume that $M_{n}$ is rational (finite Blaschke product), but $M_{d}$ and $N_{o}$ can be infinite dimensional. The relative degree of $N_{o}$ is assumed to be an integer $n_{o} \in \mathbb{N}$, i.e., we consider plants for which the decay rate of $20 \log \left(\left|N_{o}(j \omega)\right|\right)$, as $\omega \rightarrow \infty$, is $-20 n_{o} \mathrm{~dB}$ per decade, for some non-negative integer $n_{o}$.

A typical example of such plants are retarded or neutral time delay systems written in the form

$$
P(s)=\frac{R(s)}{T(s)}=\frac{\sum_{i=1}^{n_{r}} R_{i}(s) e^{-h_{i} s}}{\sum_{j=1}^{n_{t}} T_{j}(s) e^{-\tau_{j} s}}
$$

where

(i) $R_{i}$ and $T_{j}$ are stable, proper, finite dimensional transfer functions, for $i=1, \ldots, n_{r}$, and $j=1, \ldots, n_{t}$;

(ii) $R$ and $T$ have no imaginary axis zeros, but they may have finitely many zeros in $\mathbb{C}_{+}$; moreover, $T$ is allowed 
to have infinitely many zeros in $\mathbb{C}_{+}$, see below cases (ii.a) and (ii.b);

(iii) time delays, $h_{i}$ and $\tau_{j}$ are rational numbers such that $0=h_{1}<h_{2}<\ldots<h_{n_{r}} ; 0=\tau_{1}<\tau_{2}<\ldots<\tau_{n_{t}}$.

In [11] it has been shown that under the conditions given above the time delay system (4) can be put into general form (3). In order to do this, define the conjugate of $T(s)$ as $\bar{T}(s):=e^{-\tau_{n_{t}} s} T(-s) M_{C}(s)$ where $M_{C}$ is inner, finite dimensional whose poles are poles of $T$. For notational convenience, we say that $T$ is an $F$-system (respectively, $I$-system) if $T$ (respectively, $\bar{T}$ ) has finitely many zeros in $\mathbb{C}_{+}$; (note that when $T$ is an $I$-system the plant has infinitely many poles in $\mathbb{C}_{+}$). The plant factorization can be done as follows for two different cases: system:

Case (ii.a): When $R$ is an $F$-system and $T$ is an $I$ -

$$
M_{n}=M_{R}, \quad M_{d}=M_{\bar{T}} \frac{T}{\bar{T}}, \quad N_{o}=\frac{R}{M_{R}} \frac{M_{\bar{T}}}{\bar{T}},
$$

Case (ii.b): When $R$ and $T$ are both $F$-systems:

$$
M_{n}=M_{R}, \quad M_{d}=M_{T}, \quad N_{o}=\frac{R}{M_{R}} \frac{M_{T}}{T}
$$

The inner functions, $M_{R}, M_{T}$ and $M_{\bar{T}}$, are defined in such a way that their zeros are $\mathbb{C}_{+}$zeros of $R, T$ and $\bar{T}$, respectively. By assumption (ii), $R, T$ (case (ii.b)) and $\bar{T}$ (case (ii.a)) have finitely many zeros in $\mathbb{C}_{+}$, so, the inner functions, $M_{R}, M_{T}$ and $M_{\bar{T}}$ are finite dimensional.

Example. Consider a plant with infinitely many poles in $\mathbb{C}_{+}$ (this corresponds to case (ii.a) where $R$ and $T$ are $F$-system and $I$-system respectively; clearly, the plant factorization in case (ii.b) is much easier):

$$
\begin{aligned}
P_{F I}(s) & =\frac{(s+1)+4 e^{-3 s}}{(s+1)+2(s-1) e^{-2 s}} \\
& =\frac{R(s)}{T(s)}=\frac{1 e^{-0 s}+\left(\frac{4}{s+1}\right) e^{-3 s}}{1 e^{-0 s}+\left(\frac{2 s-2}{s+1}\right) e^{-2 s}} .
\end{aligned}
$$

It can be shown that $R$ has only two $\mathbb{C}_{+}$zeros at $s_{1,2} \approx$ $0.3125 \pm j 0.8548$. Also, $T$ has infinitely many $\mathbb{C}_{+}$zeros converging to $\ln \sqrt{2} \pm j\left(k+\frac{1}{2}\right) \pi$ as $k \rightarrow \infty$. This plant can be re-written as (3) with

$$
\begin{aligned}
M_{n}(s) & =\frac{\left(s-s_{1}\right)\left(s-s_{2}\right)}{\left(s+s_{1}\right)\left(s+s_{2}\right)}, \quad M_{d}(s)=\frac{T(s)}{\bar{T}(s)} \\
\bar{T}(s) & =e^{-2 s} T(-s)\left(\frac{s-1}{s+1}\right)=2+\left(\frac{s-1}{s+1}\right) e^{-2 s} \\
N_{o}(s) & =\frac{R(s)}{M_{n}(s)} \frac{1}{\bar{T}(s)},
\end{aligned}
$$

\section{Optimal Weighted Sensitivity}

In this section we illustrate how the Nevanlinna-Pick approach proposed in [8] extends to the classes of plants in the form (4). We will also see that the optimal solution in this approach leads to a non-causal optimal controller. In the next section we will modify the interpolation problem to solve this problem.
First, in order to eliminate a technical issue, which is not essential in the weighted sensitivity minimization, we will replace the outer part, $N_{o}$, of the plant with

$$
N_{\varepsilon}(s)=N_{o}(s)(1+\varepsilon s)^{n_{o}}
$$

where $\varepsilon>0$ and $\varepsilon \rightarrow 0$. This makes sure that the plant does not have a zero at $+\infty$, and hence we do not have to deal with interpolation conditions at infinity.

Now, let $s_{1}, \ldots, s_{n}$ be the zeros of $M_{n}(s)$ in $\mathbb{C}_{+}$. Then, WSMSC problem can be solved by finding a function $F(s)$ satisfying three conditions (see e.g. [6], [8], [25])

(F1) $F \in \mathcal{H}^{\infty}$ and $\|F\|_{\infty} \leq 1$;

(F2) $F$ satisfies interpolation conditions (9);

(F3) $F$ is a unit in $\mathcal{H}^{\infty}$, i.e. $F, F^{-1} \in \mathcal{H}^{\infty}$;

$$
F\left(s_{i}\right)=\frac{W\left(s_{i}\right)}{\gamma M_{d}\left(s_{i}\right)}=: \frac{\omega_{i}}{\gamma}, \quad i=1, \ldots, n .
$$

Once such an $F$ is constructed, the controller

$$
C_{\gamma}(s)=\frac{W(s)-\gamma M_{d}(s) F(s)}{\gamma M_{n}(s) F(s)} N_{\varepsilon}(s)^{-1}
$$

is in $\mathcal{S}_{\infty}(P)$ and it leads to $\left\|W(1+P C)^{-1}\right\|_{\infty} \leq \gamma$. Therefore, $\gamma_{\mathrm{ss}}$ is the smallest $\gamma$ for which there exists $F(s)$ satisfying F1, F2 and F3. It is also important to note that the controller (10) is the solution of the unrestricted weighted sensitivity minimization (WSM) problem, defined by (1), when $F(s)$ satisfies $\mathrm{F} 1$ and $\mathrm{F} 2$ for the smallest possible $\gamma>0$; in this case, since F3 may be be violated, the controller may be unstable.

The problem of constructing $F(s)$ satisfying $\mathrm{F} 1-\mathrm{F} 3$ has been solved by using the Nevanlinna-Pick interpolation as follows. First define

$$
G(s)=-\ln F(s), \quad F(s)=e^{-G(s)} .
$$

Now, we want to find an analytic function $G: \mathbb{C}_{+} \rightarrow \mathbb{C}_{+}$ such that

$$
G\left(s_{i}\right)=-\ln \omega_{i}+\ln \gamma-j 2 \pi \ell_{i}=: \nu_{i}, \quad i=1, \ldots, n
$$

where $\ell_{i}$ is a free integer due to non-unique phase of the complex logarithm. Note that when $\|F\|_{\infty} \leq 1$ the function $G$ has a positive real part hence it maps $\mathbb{C}_{+}$into $\mathbb{C}_{+}$. Let $\mathbb{D}$ denote the open unit disc, and transform the problem data from $\mathbb{C}_{+}$to $\mathbb{D}$ by using a one-to-one conformal map $z=$ $\phi(s)$. The transformed interpolation conditions are

$$
f\left(z_{i}\right)=\frac{\omega_{i}}{\gamma}, \quad i=1, \ldots, n
$$

where $z_{i}=\phi\left(s_{i}\right)$ and $f(z)=F\left(\phi^{-1}(z)\right)$. The transformed interpolation problem is to find a unit with $\|f\|_{\infty} \leq 1$ such that interpolation conditions (13) are satisfied. By the transformation $g(z)=-\ln f(z)$, the interpolation problem can be written as, $g\left(z_{i}\right)=\nu_{i}, \quad i=1, \ldots, n$. Define $\phi\left(\nu_{i}\right)=: \zeta_{i}$. If we can find an analytic function $\tilde{g}: \mathbb{D} \rightarrow \mathbb{D}$, satisfying $\tilde{g}\left(z_{i}\right)=\zeta_{i} \quad i=1, \ldots, n$, then the desired $g(z)$, hence $f(z)$ and $F(s)$ can be constructed from $g(z)=$ $\phi^{-1}(\tilde{g}(z))$. The problem of finding such $\tilde{g}$ is the well-known Nevanlinna-Pick problem, [7], [15], [26]. The condition for 
the existence of an appropriate $g$ can be given directly: there exists such an analytic function $g: \mathbb{D} \rightarrow \mathbb{C}_{+}$if and only if the Pick matrix $\mathcal{P}$,

$$
\mathcal{P}\left(\gamma,\left\{\ell_{i}, \ell_{k}\right\}\right)_{i, k}=\left[\frac{2 \ln \gamma-\ln \omega_{i}-\ln \bar{\omega}_{k}+j 2 \pi \ell_{k, i}}{1-z_{i} \bar{z}_{k}}\right]_{(14)}
$$

is positive semi-definite, where $\ell_{k, i}=\ell_{k}-\ell_{i}$ are free integers. In [8], it is mentioned that the possible integer sets $\left\{\ell_{i}, \ell_{k}\right\}$ are finite and there exists a minimum value, $\gamma_{\mathrm{ss}}$, such that $\mathcal{P}\left(\gamma_{\mathrm{ss}},\left\{\ell_{i}, \ell_{k}\right\}\right) \geq 0$.

The Nevanlinna-Pick problem posed above can be solved as outlined in [7], [15], [26]. As noted in [8], [12] and we illustrate with an example in Section $\mathrm{V}$, in general, as $\gamma$ decreases to $\gamma_{\mathrm{ss}}$ the function $G(s)$ satisfies

$$
G(s) \rightarrow k_{\gamma} s, \quad \text { where } \quad k_{\gamma} \in \mathbb{R}_{+} \text {as } s \rightarrow \infty .
$$

Therefore, in the optimal case $F(s)$ has an essential singularity at infinity, i.e., $\lim _{s \rightarrow \infty}|F(s)|=0$, thus $F^{-1}$ is not bounded in $\mathbb{C}_{+}$, i.e., $F^{-1} \notin \mathcal{H}^{\infty}$. Clearly, this violates one of the design conditions and leads to a non-causal controller (10), which typically contains a time advance. In the next section to circumvent this problem we propose to put an $\mathcal{H}^{\infty}$ norm bound on $F^{-1}$.

\section{Modified Interpolation Problem}

The controller (10) leads to the weighted sensitivity

$$
W(s)\left(1+P(s) C_{\gamma}(s)\right)^{-1}=\gamma M_{d}(s) F(s)
$$

where $F, F^{-1} \in \mathcal{H}^{\infty},\|F\|_{\infty} \leq 1$ and (9) holds. Since one of the conditions on $F$ is to have $F^{-1} \in \mathcal{H}^{\infty}$ it is natural to consider a norm bound

$$
\left\|F^{-1}\right\|_{\infty} \leq \rho
$$

for some fixed $\rho>1$. This also puts a bound on the $\mathcal{H}^{\infty}$ norm of the controller; more precisely,

$$
\left\|C_{\gamma}\right\|_{\infty} \leq\left\|N_{o}\right\|_{\infty}^{-1}\left(1+\frac{\rho}{\gamma}\|W\|_{\infty}\right) .
$$

Recall that we are looking for an $F$ in the form $F(s)=$ $e^{-G(s)}$, for some analytic $G: \mathbb{C}_{+} \rightarrow \mathbb{C}_{+}$satisfying $G\left(s_{i}\right)=\nu_{i}, i=1, \ldots, n$. In this case we will have $|F(s)|=\left|e^{-\operatorname{Re}(G(s))}\right| \leq 1$ for all $s \in \mathbb{C}_{+}$. On the other hand, $F^{-1}(s)=e^{G(s)}$. Thus, in order to satisfy (16), $G$ should have a bounded real part, namely

$$
0<\operatorname{Re}(G(s))<\ln (\rho)=: \sigma_{o}
$$

Accordingly, define $\mathbb{C}_{+}^{\sigma_{o}}:=\left\{s \in \mathbb{C}_{+}: 0<\operatorname{Re}(s)<\sigma_{o}\right\}$. Then, the analytic function $G$ we construct should take $\mathbb{C}_{+}$ into $\mathbb{C}_{+}^{\sigma_{o}}$. Note from (12) that in order for this modified problem to make sense $\gamma$ should not be too large (or $\rho$ should be large enough) so that we have a feasible interpolation data, i.e. $\nu_{i} \in \mathbb{C}_{+}^{\sigma_{o}}$. Now take a conformal map $\psi: \mathbb{C}_{+}^{\sigma_{o}} \rightarrow \mathbb{D}$, and set $\zeta_{i}:=\psi\left(\nu_{i}\right), z_{i}=\phi\left(s_{i}\right)$, where as before $\phi$ is a conformal map from $\mathbb{C}_{+}$to $\mathbb{D}$. Then, the problem is again transformed to a Nevanlinna-Pick interpolation: find an analytic function $\tilde{g}: \mathbb{D} \rightarrow \mathbb{D}$ such that $\tilde{g}\left(z_{i}\right)=\zeta_{i}$, $i=1, \ldots n$. Once $\tilde{g}$ is obtained, the function $G$ is determined as $G(s)=\psi^{-1}(\tilde{g}(\phi(s)))$. Typically, we take

$$
\begin{aligned}
z=\phi(s) & =\frac{s-1}{s+1} \quad s=\phi^{-1}(z)=\frac{1+z}{1-z} \\
z=\psi(s) & =\frac{j e^{-j \pi s / \sigma_{o}}-1}{j e^{-j \pi s / \sigma_{o}}+1} \\
s=\psi^{-1}(z) & =\frac{\sigma_{o}}{\pi}\left(\frac{\pi}{2}+j \ln \left(\frac{1+z}{1-z}\right)\right),
\end{aligned}
$$

see e.g. [17]. Interpolating functions defined above are illustrated in Figure 1.

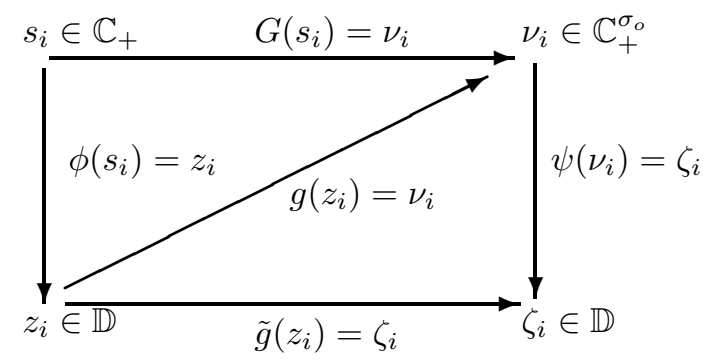

Fig. 1. Interpolating functions and conformal maps

It is interesting to note that in this modified problem $\gamma_{\mathrm{ss}}$ (smallest $\gamma$ for which a feasible $\tilde{g}$ exists) depends on $\rho$, so we write $\gamma_{\mathrm{ss}, \rho}$. As $\rho$ decreases we expect that $\gamma_{\mathrm{ss}, \rho}$ will increase; and as $\rho \rightarrow \infty$ we expect that $\gamma_{\mathrm{ss}, \rho}$ will converge to $\gamma_{\mathrm{ss}}$, the value found from the unrestricted interpolation problem summarized in Section III.

\section{EXAMPLE}

Consider the plant (7) defined earlier. Recall that it has only two $\mathbb{C}_{+}$zeros at $s_{1,2} \approx 0.3125 \pm 0.8548 j$. Let the weighting function be given as

$$
W(s)=\frac{1+0.1 s}{s+1} .
$$

Then, the interpolation conditions are $\omega_{1,2}=0.79 \mp 0.42 j$. Applying the procedure of [12], summarized in Section III, we find $\gamma_{\mathrm{ss}}=1.0704$. The optimal interpolating function is

$$
F(s)=e^{-0.57 s}
$$

and hence the optimal controller is written as

$$
C_{\gamma_{\mathrm{ss}}}=\frac{\frac{1+0.1 s}{s+1}-1.0704\left(\frac{s+1+2(s-1) e^{-2 s}}{2(s+1)+(s-1) e^{-2 s}}\right) e^{-0.57 s}}{1.0704\left(\frac{s+1+4 e^{-3 s}}{2(s+1)+(s-1) e^{-2 s}}\right) e^{-0.57 s}} .
$$

Clearly, $F^{-1} \notin \mathcal{H}^{\infty}$ and the controller is non-causal, it includes a time advance $e^{+0.57 s}$.

If we now apply the modified interpolation idea we see that as $\rho \rightarrow \infty$ the smallest $\gamma$ for which the problem is solvable, i.e. $\gamma_{\mathrm{ss}}$, approaches to 1.0704 , which is the optimal performance level found earlier. On the other hand, as $\rho$ decreases $\gamma_{\mathrm{ss}}$ increases, and there is a minimum value of $\rho=e^{0.88}=2.41$, below which there is no solution to the interpolation problem. See Figure 2. 


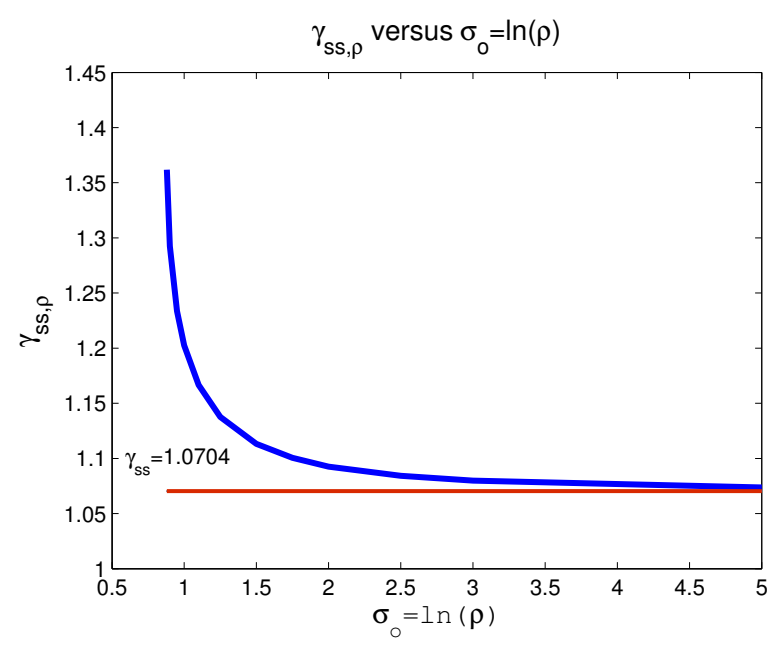

Fig. 2. $\gamma_{\mathrm{ss}, \rho}$ versus $\rho=e^{\sigma_{o}}$

For $\sigma_{o}=3$, i.e. $\rho=e^{3} \approx 20$, we have $\gamma_{\mathrm{ss}, \rho}=1.08$, and the resulting interpolant is given by

$$
\tilde{G}(s):=\tilde{g}(\phi(s))=j \frac{-0.99794(s-3.415)(s+1)}{(s+3.406)(s+1.001)} .
$$

The optimal $F(s)=e^{-G(s)}$ is determined from

$$
G(s)=\psi^{-1}(\tilde{G}(s))
$$

where $\psi^{-1}$ is as defined in (17). The optimal $F$ is

$$
F(s)=\exp \left(-\frac{\sigma_{o}}{2}-\frac{j \sigma_{o}}{\pi} \ln \left(\frac{1+\tilde{G}(s)}{1-\tilde{G}(s)}\right)\right) .
$$

Note that the optimal $F(s)$ is infinite dimensional. The magnitude and phase of $F(j \omega)$ are given in Figure 5. Rational approximations of (19) can be obtained by using model reduction techniques for stable minimum phase infinite dimensional systems.

Another way to obtain finite dimensional interpolating function $F(s)$ is to search for a proper free parameter in the set of all suboptimal solutions to the interpolation problem of finding $F$ satisfying F1-F3. For a given $\gamma>\gamma_{\mathrm{ss}}$ we can parameterize all suboptimal solutions to this problem as, (see e.g. [7])

$$
f(z)=\frac{\tilde{P}(z) q(z)+\tilde{Q}(z)}{P(z)+Q(z) q(z)}, \quad\|q\|_{\infty} \leq 1,
$$

where $\tilde{P}, \tilde{Q}, P, Q$ are computed as in [7], [15], [26]. Using first-order free parameter

$$
q(z)=\frac{a z+b}{z+c},
$$

we search for a unit $f$ in the set determined by (20). Since $\|q\|_{\infty} \leq 1$, the parameters $(a, b, c)$ are in the set

$$
\mathcal{D}_{q}:=\{(a, b, c):|c| \geq 1,|a+b| \leq|c+1|,|a-b| \leq|c-1|\} .
$$

Then a unit function $f$ can be found if there exist $(a, b, c) \in \mathcal{D}_{q}$ such that

$$
(a z+b) \tilde{P}(z)+(z+c) \tilde{Q}(z)
$$

has no zeros in $\mathbb{D}$. The problem of finding $(a, b, c)$ such that (21) has no zeros in $\mathbb{D}$ is equivalent to stabilization of discrete-time systems by first-order controllers considered in [24]. So we take the intersection of the parameters found using [24] and the set $\mathcal{D}_{q}$. The stabilization set $(a, b, c)$ is determined by fixing $c$ and obtaining the stabilization set in $a-b$ plane by checking the stability boundaries.

For the above example, let $\gamma=1.2>1.07=\gamma_{\mathrm{ss}}$. After the calculation of $\tilde{P}, \tilde{Q}, P, Q$, we obtain feasible parameter pairs $(a, b)$, for each fixed $c$, resulting in a unit $f(z)$ as shown in Figure 3. Note that all values in $(a, b, c)$ parameter set results in stable suboptimal $\mathcal{H}^{\infty}$ controller which gives flexibility in design to meet other design requirements.

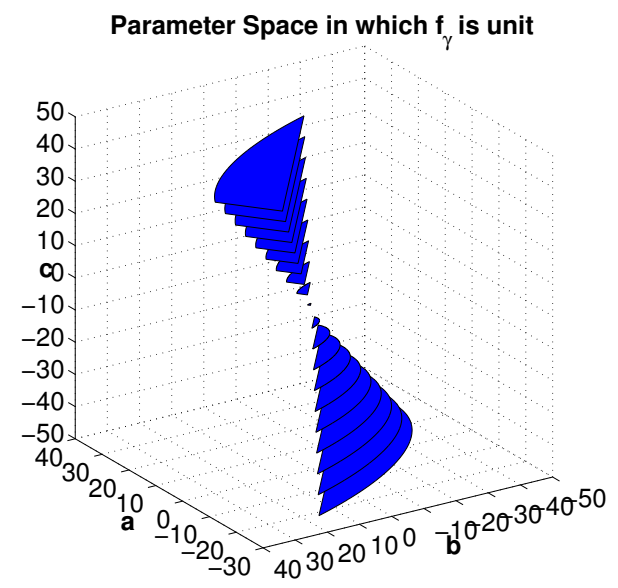

Fig. 3. Feasible $(a, b, c)$ for $f$ to be a unit.

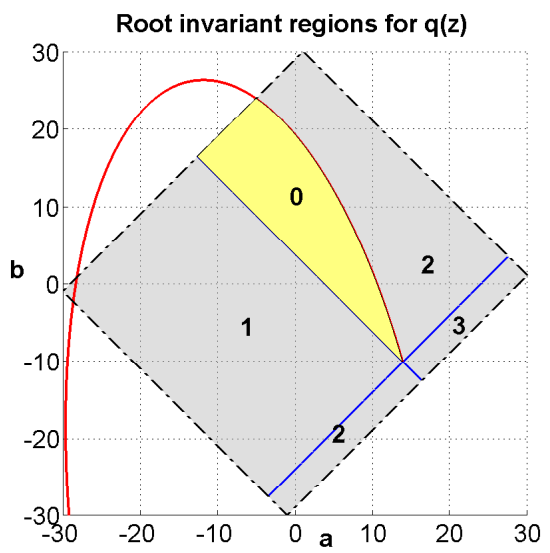

Fig. 4. Root invariant regions for $c=30$.

In Figure 4, stability region for (21) is given for $c=$ 30 . Red and blue lines are real and complex-root crossing boundaries respectively. The yellow colored region (labeled as region 0 in the grayscale print) is where the polynomial (21) has no $\mathbb{C}_{+}$zero and the corresponding $\mathcal{H}^{\infty}$ controller is stable. The value of $\gamma=1.2$ is chosen to show the controller parameterization set and stability regions clearly. If we apply the same technique for $\gamma=1.08$ the feasible region in $\mathbb{R}^{3}$ shrinks, but we still get a solution:

$$
F(s)=\frac{0.068 s^{3}+3.77 s^{2}+21.45 s+295.84}{9.93 s^{3}+62.77 s^{2}+187.25 s+296.27} .
$$


It is easy to verify that

$$
F\left(s_{i}\right)=\frac{\omega_{i}}{1.08}, \quad \text { for } i=1,2 .
$$

The function $F$ is a unit with poles and zeros

$$
\begin{aligned}
& \operatorname{zero}(\mathrm{F})=-50.9245,-2.2583 \pm j 8.9628 \\
& \operatorname{pole}(\mathrm{F})=-3.3510,-1.4851 \pm j 2.5881
\end{aligned}
$$

and from its Bode plot we find $\|F\|_{\infty}=\frac{295.84}{296.27}<1$. Moreover, $F^{-1} \in \mathcal{H}^{\infty}$ with $\left\|F^{-1}\right\|_{\infty} \approx 146$.

In order to compare the third order $F$ given in (22), with the infinite dimensional $F$ given in (19), (both of them are designed for $\gamma=1.08$ ) we provide their magnitude and phase plots in Figure 5.
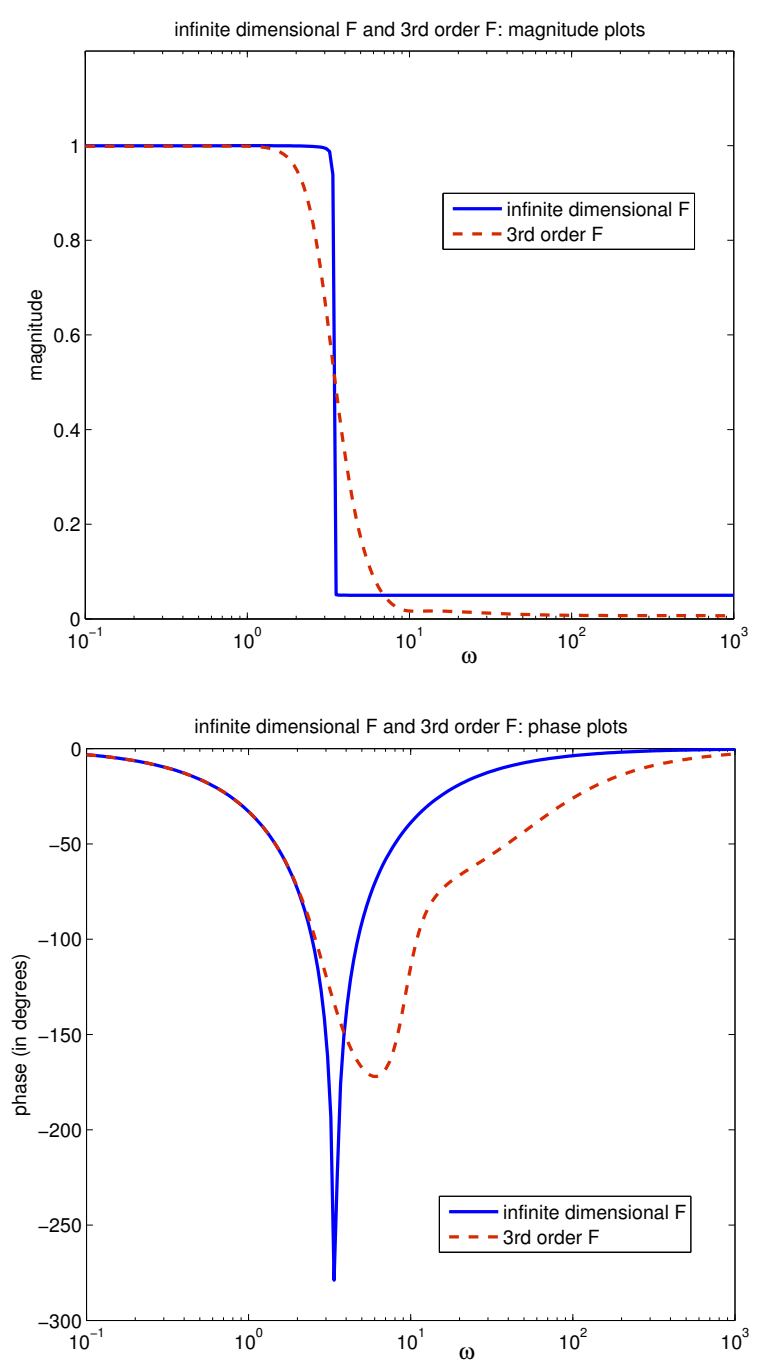

Fig. 5. Magnitude and phase plots of $F$ given in (19) and (22).

Although finding a finite dimensional $F(s)$ results in infinite dimensional suboptimal controller $C_{\gamma}(s),(10)$, it is possible to implement the controller in a stable manner as illustrated below. Recall that the controller is in the form

$$
C_{\gamma}(s)=\left(\frac{\gamma^{-1} F^{-1}(s) W(s)-M_{d}(s)}{M_{n}(s)}\right) N_{\varepsilon}^{-1}(s) .
$$

Note that there are unstable pole-zero cancellations inside the parenthesis in the above expression, and in $N_{\varepsilon}$. The unstable pole-zero cancellations for this system can be avoided by the method proposed in [11] as follows:

$$
\begin{aligned}
C_{\gamma} & =\left(\frac{\gamma^{-1} F^{-1}(s) W(s) \bar{T}(s)-M_{\bar{T}}(s) T(s)}{M_{R}(s) M_{\bar{T}}(s)}\right) \frac{M_{R}(s)}{R(s)} \\
& =\left(H_{T}(s)+\mathcal{F}_{T}(s)\right)\left(H_{R}(s)+\mathcal{F}_{R}(s)\right)^{-1}
\end{aligned}
$$

where $\mathcal{F}_{T}$ and $\mathcal{F}_{R}$ are finite impulse response filters (i.e. their impulse responses are non-zero only on a finite time interval)

$$
\begin{aligned}
& \mathcal{F}_{R} \approx \frac{1.25 s+(2.04 s+1.69) e^{-3 s}}{s^{2}-0.625 s+0.828} \\
& \mathcal{F}_{T} \approx \frac{0.585 s+0.019-(0.285 s-1.066) e^{-2 s}}{s^{2}-0.625 s+0.828}
\end{aligned}
$$

whose denominators are determined from the zeros of $M_{n}$. The impulse responses of $\mathcal{F}_{T}$ and $\mathcal{F}_{R}$ are given in Figure 6. The terms, $H_{R}$ and $H_{T}$, are time-delay systems with no unstable pole-zero cancellations internally.
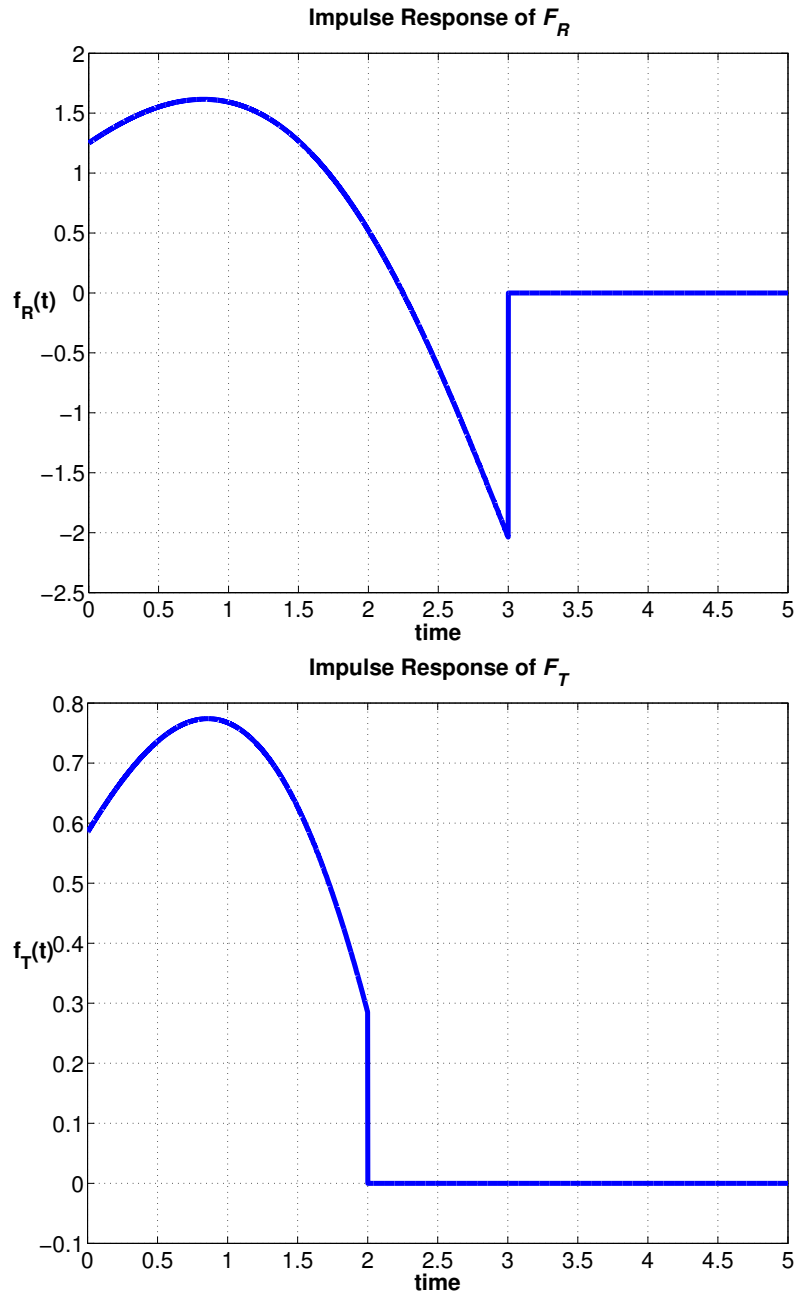

Fig. 6. Impulse Responses of $\mathcal{F}_{R}$ and $\mathcal{F}_{T}$ 


\section{CONCLUSIONS}

In this note we have modified the Nevanlinna-Pick interpolation problem appearing in the computation of the optimal strongly stabilizing controller minimizing the weighted sensitivity. By putting a bound on the norm of $F^{-1}$, a bound on the $\mathcal{H}^{\infty}$ norm of the controller can be obtained. We have obtained the optimal $\gamma_{\mathrm{ss}, \rho}$ as a function of $\rho$, where $\left\|F^{-1}\right\|_{\infty} \leq \rho$. The example illustrated that as $\rho \rightarrow \infty$, $\gamma_{\mathrm{ss}, \rho}$ converges to the optimal $\gamma_{\mathrm{ss}}$ for the problem where $\left\|F^{-1}\right\|_{\infty}$ is not constrained. The controller obtained here is again infinite dimensional; for practical purposes it needs to be approximated by a rational function. In general this method may require very high order approximations since the order of strongly stabilizing controllers for a given plant (even in the finite dimensional case) may have to be very large, [22].

An alternative method for finding a low order $F$ satisfying all conditions is also illustrated with the given example. It searches for a first order free parameter leading to a unit $f$. Note that even when $F$ is finite dimensional, the controller is still infinite dimensional. We have also illustrated how this infinite dimensional controller can be implemented in a stable manner by decomposing it into parts which contain no unstable pole-zero cancelations. Then these individual terms can be approximated in a stable manner to find a finite dimensional controller.

\section{REFERENCES}

[1] A. E. Barabanov, "Design of $\mathcal{H}^{\infty}$ optimal stable controller," Proc. IEEE Conference on Decision and Control, pp. 734-738, 1996.

[2] D. U. Campos-Delgado and K. Zhou, "H户 Strong stabilization," IEEE Transactions on Automatic Control, vol.46, pp. 1968-1972, 2001.

[3] D. U. Campos-Delgado and K. Zhou, "A parametric optimization approach to $\mathcal{H}^{\infty}$ and $\mathcal{H}^{2}$ strong stabilizaiton," Automatica, vol. 39, No. 7, pp. 1205-1211, 2003.

[4] Y. Choi and W.K. Chung, "On the Stable $\mathcal{H}^{\infty}$ Controller Parameterization Under Sufficient Condition," IEEE Transactions on Automatic Control, vol.46, pp. 1618-1623, 2001.

[5] Y.S. Chou, T.Z. Wu and J.L. Leu, "On Strong Stabilization and $\mathcal{H}^{\infty}$ Strong-Stabilization Problems," Proc. Conference on Decision and Control, pp. 5155-5160, 2003.

[6] J. C. Doyle, B. A. Francis, A. R. Tannenbaum, Feedback Control Theory, Macmillan, NY, 1992.

[7] C. Foias, H. Özbay, A. Tannenbaum, Robust Control of Infinite Dimensional Systems: Frequency Domain Methods, Lecture Notes in Control and Information Sciences, No. 209, Springer-Verlag, London, 1996.

[8] C. Ganesh and J. B. Pearson, "Design of optimal control systems with stable feedback," Proc. American Control Conf., pp. 1969-1973, 1986.

[9] S. Gümüsssoy and H. Özbay, "On Stable $\mathcal{H}^{\infty}$ Controllers for TimeDelay Systems," in Proceedings of the 16th Mathematical Theory of Network and Systems, Leuven, Belgium, July 2004.

[10] S. Gümüşsoy, and H. Özbay, "Remarks on Strong Stabilization and Stable $\mathcal{H}^{\infty}$ Controller Design," IEEE Trans. on Automatic Control, vol. 50, pp. 2083-2087, 2005.

[11] S. Gümüşsoy and H. Özbay, "Remarks on $\mathcal{H}^{\infty}$ Controller Design for SISO Plants with Time Delays," in the proceedings of the 5th IFAC Symposium on Robust Control Design, Toulouse, France, July, 2006.

[12] S. Gümüşsoy and H. Özbay, "Sensitivity Minimization by Stable Controllers for a Class of Unstable Time-Delay Systems" Proc. of the 9th International Conference on Control, Automation, Robotics and Vision (ICARCV 2006), Singapore, pp. 2161-2165, December 2006.

[13] H. Ito, H. Ohmori and A. Sano, "Design of stable controllers attaining low $\mathcal{H}^{\infty}$ weighted sensitivity," IEEE Trans. on Automatic Control, vol.38, pp. 485-488, 1993.
[14] M. Jacobus, M. Jamshidi, C. Abdullah, P. Dorato and D. Bernstein, "Suboptimal strong stabilization using fixed-order dynamic compensation," Proc. American Control Conference, pp. 2659-2660, 1990.

[15] M. G. Krein and A. A. Nudel'man The Markov Moment Problem and Extremal Problems, Translations of Mathematical Monographs, Vol. 50, AMS, 1977.

[16] P.H. Lee and Y.C. Soh, "Synthesis of stable $\mathcal{H}^{\infty}$ controller via the chain scattering framework," System and Control Letters, vol.46, pp. 1968-1972, 2002.

[17] Z. Nehari, Conformal Mapping, Dover, NY, 1975 (reprint of the 1952 edition).

[18] I. Petersen, "Robust $\mathcal{H}^{\infty}$ control of an uncertain system via a stable output feedback controller," Proc. American Control Conference, pp. 5000-5007, 2006

[19] I. Petersen, "Robust $\mathcal{H}^{\infty}$ control of an uncertain system via a strict bounded real output feedback controller," Proc. of the 45th IEEE Conference on Decision \& Control San Diego, CA, pp. 571-577, 2006.

[20] A.A. Saif, D. Gu and I. Postlethwaite, "Strong stabilization of MIMO systems via $\mathcal{H}^{\infty}$ optimization," System and Control Letters, vol.32, pp. 111-120, 1997.

[21] A. Sideris and M. G. Safonov, "Infinity-norm optimization with a stable controller," Proc. American Control Conference, pp. 804-805, 1985.

[22] M. C. Smith, and K. P. Sondergeld, "On the order of stable compensators," Automatica, vol. 22, pp. 127-129, 1986

[23] K. Suyama, "Strong stabilization of systems with time-delays," Proc. IEEE Industrial Electronics Society Conference, pp. 1758-1763, 1991

[24] R.N. Tantaris, L.H. Keel and S.P. Bhattacharya, "Stabilization of Discrete-Time Systems by First-Order Controllers," IEEE Transactions on Automatic Control, vol.48, pp. 858-861, 2003.

[25] M. Vidyasagar, Control System Synthesis: A Factorization Approach, MIT Press, 1985

[26] M. Zeren and H. Özbay, "Comments on 'Solutions to Combined Sensitivity and Complementary Sensitivity Problem in Control Systems',' IEEE Transactions on Automatic Control, vol.43, p. 724, 1998.

[27] M. Zeren and H. Özbay, "On the synthesis of stable $\mathcal{H}^{\infty}$ controllers," IEEE Transactions on Automatic Control, vol.44, pp. 431-435, 1999.

[28] M. Zeren and H. Özbay, "On the strong stabilization and stable $\mathcal{H}^{\infty}$. controller design problems for MIMO systems," Automatica, vol.36, pp. 1675-1684, 2000. 